\title{
Site Protection Efforts at the AURA Observatory in Chile
}

\author{
R. Chris Smith ${ }^{1}$, Pedro Sanhueza ${ }^{2}$ and Malcolm G. Smith ${ }^{3}$ \\ ${ }^{1}$ AURA Observatory in Chile, \\ Avenida Juan Cisternas 1600, La Serena, Chile \\ email: csmith@aura-o.aura-astronomy.org \\ ${ }^{2} \mathrm{OPCC}$, \\ Avenida Juan Cisternas 1606, La Serena, Chile \\ email: psanhueza2007@gmail.com \\ ${ }^{3} \mathrm{NOAO} / \mathrm{CTIO}$, \\ Avenida Juan Cisternas 1600, La Serena, Chile \\ email: msmith@ctio.noao.edu
}

\begin{abstract}
The AURA Observatory site in northern Chile, which includes Cerro Tololo and Cerro Pachon, has been operational for over 50 years now, facing a variety of challenges to its long-term future. The site now hosts over 20 operational telescopes, ranging from small projects with $0.4 \mathrm{~m}$ telescopes to the Blanco $4 \mathrm{~m}$, the SOAR $4.1 \mathrm{~m}$, and the $8 \mathrm{~m}$ Gemini-South telescopes. In addition, we have recently begun the construction of the Large Synoptic Survey Telescope (LSST) on the summit of Cerro Pachon. We summarize our efforts over the past 20-30 years to highlight the importance of site protection through education and public outreach as well as through more recent promotion of IDA certifications in the region and support for the World Heritage initiatives described by others in this conference.
\end{abstract}

Keywords. atmospheric effects, site testing

\section{Overview}

The AURA Observatory in Chile (AURA-O) was the first of the major international observatories to be established in northern Chile to exploit the optimal astronomical conditions available there. The site was originally established in 1962 to host the Cerro Tololo Inter-American Observatory (CTIO). It now hosts more than 20 operational telescopes, including some of the leading U.S. and international astronomical facilities in the southern hemisphere, such as the $4 \mathrm{~m}$ Blanco telescope on Cerro Tololo and the $4 \mathrm{~m}$ SOAR and $8 \mathrm{~m}$ Gemini-South telescopes on Cerro Pachon. Construction of the next generation facility, the Large Synoptic Survey Telescope (LSST), has recently begun on Cerro Pachn, while additional smaller telescopes continue to be added to the complement of facilities on Cerro Tololo.

While the site has become a major platform for international astronomical facilities over the last 50 years, development in the region has led to an ever-increasing threat of light pollution around the site. AURA-O has worked closely with local, regional, and national authorities and institutions (in particular with the Chilean Ministries of Environment and Foreign Relations) in an effort to protect the site from threats so that future generations of telescopes, as well as future generations of Chileans, can benefit from the dark skies in the region. In the following we briefly describe our site protection efforts, starting with the protectin of the site from mining interests and moving to protection from light pollution. 


\section{Outstanding Sites \& Evolution of Protection}

Northern Chile hosts some of the best sites in the world for astronomical groundbased facilities. The combination of high mountains, one of the driest deserts in the world, and the smooth air flow coming off the Pacific provides excellent conditions for optical, infra-red, and radio observations. In addition to these natural conditions, the solid infrastructure and stable economic and political conditions of Chile allow international observatories to operate efficiently and effectively plan for long-term investments.

In the late 1950s, Dr. Frederico Rutllant, then Director of the Chilean National Observatory, noted the development of the next generation of major observatories in the Northern hemisphere and recognized the opportunity that the conditions in northern Chile provided for astronomy. He traveled to the United States and visited key figures in the development of new astronomical facilities there, inviting them to consider establishing one or more astronomical observatories in Chile. This initiative led to the selection of Cerro Tololo as the site for the first major international observatory in Chile in November of 1962. The observatory was named the Cerro Tololo Inter-American Observatory, or CTIO, in recognition of the collaborative nature of the initiative.

Although atmospheric conditions over northern Chile provide outstanding conditions for astronomical observations, the geology has provided a much more tangible benefit for the Chilean economy. Northern Chile is rich in copper, gold, and other valuable minerals, and the mining industry has been the main economic engine for Chile for many decades. As such, mining poses a significant threat to the astronomical facilities sited in northern Chile. The Chilean government recognized this threat, and developed a mechanism for protecting the sensitive areas around the many observatories that have come to Chile since the 1960s. The sites have been declared "areas of scientific interest", a designation that requires approval by the President of Chile for any mining activity within the areas.

The rapid economic development during the 1980s and 1990s in northern Chile brought a new threat to the forefront, that of the lights of the cities in the region around the observatories. In the late 1990s, AURA-O and the other international observatories worked with the Chilean government to highlight the threat and look for ways to mitigate it while not interfering with the economic development in the region. The result was the first "norma", or regulation, on light pollution, published in 1998 as Decreto Supremo 686. In addition, the "Oficina de Protecion de la Calidad de los Cielos del Norte de Chile", or OPCC, was established to support the implementation of the norma through education and support of municipalities in the selection of appropriate public lighting.

\section{Education \& Public Outreach initiatives}

Following in the pattern developed by OPCC of stressing education and support of the community, as opposed to an emphasis on enforcement, AURA-O has developed a multi-faceted approach to raising the awareness of the impacts of light pollution and the importance of protecting the dark skies of northern Chile. Through its divisions of CTIO, Gemini, and more recently LSST, the Observatory has developed a variety of educational and public outreach (EPO) programs to carry the messages into the community.

NOAO/CTIO: The EPO program of CTIO consists of a diverse set of activities in the community, with an emphasis on training teachers and other community educators, both in formal and informal settings. The strategy is to take advantage of the multiplicative effect that one educator may have, touching hundreds if not thousands of students and/or public. Together with this training, NOAO's EPO experts have developed a variety of educational kits for the classroom and other events, including the Galileoscopes (for the 
International Year of Astronomy), Dark Sky Awareness kits, and most recently a Quality of Light kit for the International Year of Light.

Gemini: The Public Information Office (PIO) of Gemini also supports a variety of community outreach, emphasizing sharing the wonders of the Universe through major public events. Gemini sponsors two large events annually, AstroDay and Viaje al Universo, touching thousands of public participants with each event. Messages of appreciation of dark skies and the impacts of light pollution are woven into these events to sensitize the community about these important issues.

LSST: The LSST is the newest of AURA's initiatives in Chile, and with it comes a new perspective on outreach, one of public participation in the science. LSST is developing a strong "Citizen Science" program in which the public can browse through the petabytes of images that LSST will take each year and help classify galaxies, stars, and other celestial objects and events. Through these programs, AURA and LSST hope to make the Universe more accessible to the public, and in doing so also help them understand the importance of protecting this special window to the Universe that Chile provides.

In addition to these focused EPO programs, AURA has supported initiatives to create municipal and touristic observatories in the region. What started as an idea for the first municipal observatory in the nearby community of Vicuna has developed into a growing industry of "astro-tourism" in northern Chile! The industry now includes not only touristic observatories, but has spread to many of the nearby hotels (providing telescopes and nighttime programs) as well as nighttime activities such as guided horseback rides with astronomical observations included.

\section{Path Forward}

Through collaborative efforts with the Chilean government at local, regional, and national levels, we have made substantial progress in protecting the astronomical sites in northern Chile from both mining and the ever-growing issue of light pollution. In 2013 the Chilean government issued updated regulations for outdoor lighting in the region, and those regulations are beginning to be implemented as of this writing.

Beyond these regulations, the AURA-O and the other international observatories, including the European Southern Observatory, Carnegie Observatory, and the Giant Magellan Telescope, continue to strengthen their ties with the Chilean community through educational programs and outreach activities, in an effort to both get the public excited about the science and the wonders of the Universe while also sensitize them to the fragile condition of the dark skies and the threats those skies face. Most recently the AURA-O site was recognized as the first international Dark Sky Sanctuary by the International Dark-Sky Association, in recognition of the importance of the dark skies in northern Chile and the efforts to protect them. These efforts of international recognition are being furthered by the Chilean government through its initiative to propose key astronomical sites in northern Chile as UNESCO World Heritage sites. We all hope that through these initiatives and collaborations, we can ensure that the skies of northern Chile remain dark for decades, if not centuries, to come for the enjoyment and wonder of future generations of astronomers as well as children of all ages. 